\title{
PERFORMANCE OF CORE METHODOLOGIES FOR CONSUMER PRICE PREDICTION SERVICES
}

\author{
Anne Falkenberg \\ Catholic University of Eichstaett-Ingolstadt, Auf der Schanz, 49, 85049 Ingolstadt, Germany
}

\begin{abstract}
The need to monitor prices gave rise to price comparison sites that have not yet adopted recommendation services to support customers in scheduling buying decisions. Using a large data set with 1.46 million daily minimum price observations for four product categories of electronic consumer goods, we outline reasons for the slow adoption of recommendation services, evaluate almost 1.1 billion price forecasts, and show that product prices are forecastable. We compare 16 different methods that can act as the core of price prediction services and give detailed insights on performance as well as advice on model selection for different product categories and advocate the transition towards prescriptive price analytics services.
\end{abstract}

\section{KEYWORDS}

Price Forecasting, Big Data, e-Commerce, Price Time Series, Predictive Analytics

\section{INTRODUCTION}

Consumers increasingly adopt online and mobile retail channels, which not only offer more convenient shopping experiences but also increase price transparency. Despite the universal availability of price information that allows comparing different offers, this also leads to a large amount of available prices especially for standardized products (Pan et al., 2002). Therefore, a key element of modern retail business models are pricing strategies that critically shape retailers' margins and profits (Bolton et al., 2006). Because price changes in traditional channels were rather rare, now that products are available online, pricing receives much more attention and price-setting behavior becomes more dynamic (Kannan et al., 2001; Kopalle et al., 2009; Levy et al., 2004). Price changes partially follow rational patterns and prices are adjusted based on demand and inventory considerations or as differentiation from other e-commerce platforms. Smaller or specialized retailers however often follow opportunistic patterns where the objectives are not transparent or price changing actions are not guided by algorithms. This uncertainty for the customer in combination with their high price sensitivity especially for homogeneous consumer goods gave rise to price aggregation platforms that act as a facilitator and allow customers to easily gain an overview of the retail landscape.

Apart from static or historical information, price comparison sites (PCS) like idealo or shopbrain recently started offering services to actively support customers in the buying process and enable them to use the provided information to optimize their purchase time decisions. The core of these services is usually a methodology to extrapolate prices based on historical information that allows condensing expectations about future price developments. However, compared to predictive or prescriptive analytics services found in the airfare industry, the approaches observable in the consumer goods retail market are quite elementary. While it seems surprising that there is such a difference between the airfare and consumer goods industries, this can be at least partially explained by characteristics of the underlying data generation process, the available data and, therefore, methodologies disposable to solve the underlying forecasting problem.

Given a product that a customer wants to purchase, s/he is usually not interested in the price from one specific seller. If a customer is not willing to choose from a range of quality-controlled sellers but sticks to one retailer, there will be no need for her/him to actually monitor the retail landscape via an aggregation platform. The entire business model of PCS, therefore, grounds on the users' flexibility about the place of purchase. The price of an individual retailer alone is no longer of primary interest to the customer. Instead, the minimum of all prices from the listed retailers constitutes the relevant reference price. The corresponding daily minimum prices are crucial to the customer and represent what PCS usually display in their historical pricing charts. 
This process generates price time series with specific characteristics. Prices of consumer goods usually deteriorate over time, thus exhibit a time-dependent level. Price adjustments occur irregularly and with varying magnitude leading to calmer and more active price changing phases as well as to entirely constant segments. Time series of minimum prices exhibit similar characteristics than the ones from individual sellers but show more extreme values and shorter, more irregular segments with constant prices. When analyzing day-to-day price movements and thus the sequence of price changes, these constant segments lead to many observations that are zero, which is referred to as zero-inflation (Kömm and Küsters, 2015; Winkelmann, 2008). Many traditional forecasting techniques assume a strictly continuous distribution of the forecasted data (Hyndman and Athanasopoulos, 2018). This assumption is violated in the given case. However, methodological approaches that explicitly consider these shortcomings are rare, computational cumbersome and thus not scalable or directly applicable to forecast durable consumer goods prices (Buchwitz and Küsters, 2018; Kömm and Küsters, 2015; Rydberg and Shephard, 2003; Sucarrat and Grønneberg, 2016). The nature of the forecasting setting, as well as different challenges evolving from the concerning industry, customer environment and the time series characteristics, make forecasting minimum price time series for multiple product categories demanding. However, it is necessary if PCS want to enhance their service to help customers optimize their purchase time decisions.

In this paper, we aim to investigate the forecasting performance of available and scalable forecasting techniques for analytics services when applied to composed minimum price time series of consumer goods. In doing so, we evaluate and compare 16 methods for a forecast horizon of 8 weeks exemplary on 4 product groups: refrigerators, computer memory, graphic cards and smartphones. We focus on this short-term forecast horizon for two reasons: First, it is the most relevant for customers' buying decisions. Second, notification services like price alarms have an average waiting time up to two months for a $5 \%$ price reduction independent of the product category, showing that this time period is highly relevant for practical implementation (idealo, 2017). Our research is to the best of our knowledge the first contribution that benchmarks forecasting performance of core methodologies for prices of consumer durable goods for a broad range of product categories.

\section{DATA SET}

The analysis is based on a sample of daily minimum price observations from the German e-commerce market for 2,000 electronic consumer goods ranging from home appliances over computer hardware to smartphones, equally split into four categories. To make results comparable, we focus on the length of a typical life cycle for electronic consumer products and analyze the first two years of data resulting in 730 observations per item. In total, this yields a data set with 1.46 million daily observations. All items stem from well-known and established brands and each time series represents a specific entity with completely homogeneous properties and features, meaning different product configurations, sizes or colors constitute different time series.

Table 1. Descriptive statistics by product category

\begin{tabular}{|l|c|c|c|c|c|}
\hline Category & $\begin{array}{c}\text { Avg. Initial } \\
\text { Price in } €\end{array}$ & $\begin{array}{c}\text { Average } \\
\text { Price in } €\end{array}$ & $\begin{array}{c}\text { Average Daily } \\
\text { Price Change in } €\end{array}$ & $\begin{array}{c}\text { \% of Constant } \\
\text { Observations } \\
\text { (zero-inflation) }\end{array}$ & N \\
\hline Computer Memory & 237.47 & 145.32 & -0.17 & $38 \%$ & 500 \\
\hline Graphic Cards & 279.86 & 235.14 & -0.05 & $45 \%$ & 500 \\
\hline Refrigerators & 829.04 & 709.79 & -0.19 & $72 \%$ & 500 \\
\hline Smartphones & 496.71 & 333.29 & -0.29 & $64 \%$ & 500 \\
\hline All & 460.77 & 355.89 & -0.18 & $55 \%$ & 2,000 \\
\hline
\end{tabular}

Table 1 provides the descriptive statistics for the nominal price level and the price changing frequency for the four product categories. The products with the highest average initial price are refrigerators with $829.04 €$ directly followed by smartphones with $496.71 €$. However, the single highest initial price with roughly $3,900 €$ comes from an AMD Graphic Card for Professional Workstations. Interestingly, the average daily price change is not directly linked to the price level so that computer memories reach an average daily price reduction of $0.17 €$, compared to graphic cards that only deteriorate with a rate of $0.05 €$ per day-to-day price movement. 
The average daily price change of all products in the sample amounts to $-0.18 €$. The most volatile products in terms of occurring price changes are memory modules, where only $38 \%$ of the observations do not change compared to the price from the previous day. Compared to this, refrigerator prices are more constant. With $72 \%$ zero-inflation in the data, the minimum price for a refrigerator changes on average about twice a week.

Figure 1 provides an aggregated overview of the utilized data. Each of the figures (1a-1d) shows the average relative price development over the first two years of the product life cycle for the respective category. Given a single time series $\left\{y_{t}\right\}_{t \in \mathbb{N}}$, each daily price $y_{t}$ is divided by the initial price $y_{1}$ to obtain the relative price. The solid black line displays the relative price averaged over all products of the respective category. The shaded areas around the solid black line correspond to the 50\%, 75\% and $90 \%$ empirical confidence intervals of the relative prices. On average, all prices decrease over time but show different intensities of price deterioration. Besides, the longer the products are in the market, the wider the confidence intervals become. While this is nicely illustrated in figures $1 \mathrm{c}$ and $1 \mathrm{~d}$, for refrigerators the intervals stay almost constant independent of the products' time in the market. On average, the price level for the home appliance products (1a) drops rapidly but the steepness quickly decreases with the price level before stabilizing at around $85 \%$ of their starting price. While this is comparable to the price development of graphic cards shown in figure 1c, the average price level here starts to slightly increase at the end of the life cycle. Graphic cards show an identifiable global minimum approximately 1.5 years after their product launch, which cannot be observed in the other three categories.
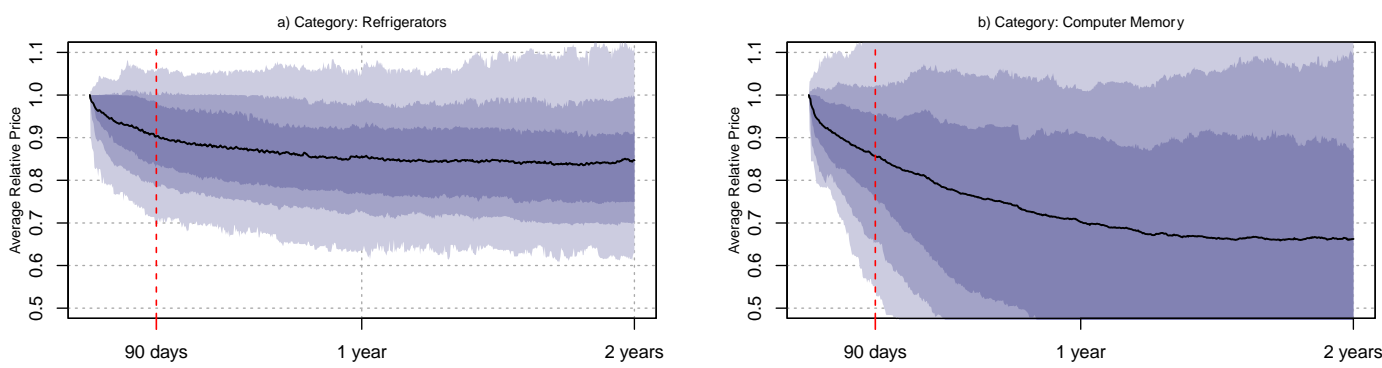

c) Category: Graphics Cards
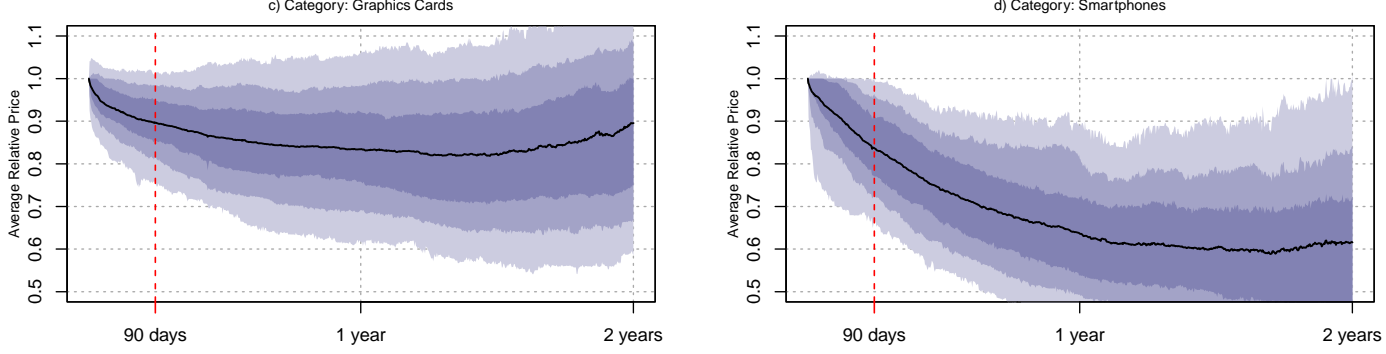

Figure 1. Relative price developments for each product category.

Both - computer memory modules and smartphones - show a comparably strong price deterioration. Even though their respective slope also decreases with the level, computer memory prices stabilize at around $65 \%$ of the starting price as shown in figure $1 \mathrm{~b}$. The settlement point for the smartphone product category is even lower with around $60 \%$. However, while the general price dynamic of these two categories seem comparable, computer memories exhibit their own momentum. The intervals shown in figure $1 \mathrm{~b}$ are almost three times wider than the ones shown in the remaining graphs despite representing the same quantiles. This indicates strong heterogeneity between individual items and may also be connected to the low zero-inflation within this category. Summing up the average price development, one can state that the overall shape is comparable for all average relative prices as each category shows deterioration, however, due to variations in slope and settlement levels, the average appearance varies.

\section{METHODOLOGY}

As shown by the descriptive statistics, forecasting approaches have to deal with a variety of paradigms that are present in the data. Besides the non-transparent data generation process, this includes time series with rapidly 
changing dynamics, potentially time-varying mean structures and local up- or downward movements that overlay the global price development. Due to this variety, it is unrealistic to expect a single method to deliver the best forecasts independent of these specific characteristics and independent of the forecast horizon. We, therefore, choose methodological corpora that possess different functionalities to deal with the features in the price data.

\subsection{Forecasting Models}

As several of the selected approaches belong to the same model families, represent simple or special cases of modified models or can be differentiated by pre- and post-processing options, the approaches can be grouped into the following seven classes. Due to the restricted length of this article, the technical details are not explained in-depth but cited accordingly. For each class, we focus on two to four configuration options, which leads to a selection of 16 methods that we elaborate on in the upcoming results section.

Global and Local Trend: Given the fact that the majority of electronic consumer product prices deteriorate over time, models that assume a global trend, present an intuitive benchmark for more complex approaches. We, therefore, include linear time series regression models (Makridakis et al., 1997) that are estimated using ordinary least squares as a benchmark. While the simplest form models a global linear trend (Trend), we also generate forecasts using an exponential trend (Trend exp.) and employ a configuration that contains a quadratic term (Trend sq.) to account for different shapes of price deterioration. As the data contains local dynamics, it may be plausible to restrict the amount of data used for the trend extrapolation to make the model more adaptive to local changes. For this reason, we generate forecasts based on a quarterly sliding window (Trend 90).

Mean Models formulate expectations for future price developments based on the arithmetic mean and, therefore, assume that deviations from the mean are based on random perturbations. Following the approach applied by $e$ Bay to signal price tendencies to their users, we include a global mean model (Mean) and the arithmetic mean based on the last 90 observations (Mean 90) in our comparison. Both trend and mean models together represent the forecasting approaches known to be currently in place and observable in practice and are, therefore, used mainly as a benchmark for more complex approaches.

Random Walk: Assuming the difference between two consecutive price observations is white noise, pure random walk models $(R W)$ use the last available observation as the forecast for all future horizons. While mean and random walk models implement two divergent principles, the mean forecast converges towards a random walk prediction with increasing zero-inflation. Given that there are multiple constant segments in the price time series and that prices show strong price deterioration, we enhance the random walk with a drift term $(R W$ Drift) (Hyndman and Athanasopoulos, 2018). This allows the model to incorporate a global tendency while eradicating some shortcomings of the presented trend approaches.

ARIMA: Autoregressive Integrated Moving Average models (ARIMA) depict the inherent dynamics in the price time series and therefore represent a more adaptive group of forecasting approaches. While such models are often described as short-term oriented, this is especially true for types that assume a stationary time series (Box et al., 1994). However, ARIMA models can also be applied to time series originating from an instationary data generation process and include coefficients to incorporate longer-term tendencies in the data. A fitted slope reflects the inherent direction of the price development after accounting for the partial autocorrelation (moving average) and autoregressive structure in the data. To estimate models, a concrete specification has to be determined, for which we revert to a procedure that automatically selects the model order that fits best to the price data (AutoARIMA) (Hyndman and Khandakar, 2008). This setting considers stationary as well as instationary model configurations and includes an appropriate number of autoregressive and moving average coefficients to capture the price dynamics. Additionally, we also employ forecasts that originated from models assuming instationary data generation processes, so that only the autoregressive and moving average orders are automatically selected (AutoARIMA inst.).

Exponential Smoothing: The inherent modeling idea of exponential smoothing models (EXS) is that forecasts are adjusted based on a local and time-varying level, trend and/or error estimates, while the speed of the adjustment is controlled via smoothing constants. Some model configurations allow for additive or multiplicative components in each case with or without damped trends (Holt, 2004) or combine these approaches with additive or multiplicative seasonality indices. An overview of the existing and considered configurations is given by Pegels (1969) and Gardner (2006, 1985). To estimate and forecast exponential smoothing models, we refer to their state-space representation that also enables automatic model selection 
(EXS) (Hyndman et al., 2008). However, due to the good results in forecasting competitions (Makridakis and Hibon, 2000), we include some pure models in addition to the automated model selection approach - most importantly an exponential smoothing model with additive, damped trend (EXS dHolt). This allows us to derive conclusions based on the different components considered by the models.

Theta: The Theta method is a decomposition approach where the segmented components of a time series are called Theta-lines (Assimakopoulos and Nikolopoulos, 2000). Hyndman and Billah (2003) show that the generated forecasts are numerically equivalent to an exponential smoothing model combined with a drift term. This drift coefficient is equal to half of the slope coefficient when fitting a linear regression model to the data. For this reason, the Theta method is known to generate conservative predictions that show no extreme up- or downward tendencies. We employ an adapted version of the Theta method (FourTheta) (Nikolopoulos and Assimakopoulos, 2004) that is more tailored to the data in addition to the original version that we present in combination with a logarithmic transformation of the prices (logTheta) (Assimakopoulos and Nikolopoulos, 2000; Box and Cox, 1964).

Combination Methods have the potential to eradicate shortcomings from the individual methods through calculating the (weighted) average of the derived point forecasts (Clemen, 1989). Obviously, the resulting performance depends on the incorporated method. We consider static and equally weighted combinations for the automatic selection procedures of EXS and ARIMA models (Combi EA) as well as for a group of exponential smoothing models consisting of the simple exponential smoothing and exponential smoothing with and without damped trend (Combi SHD) that was also used in the M4 forecasting competition (M4 Competition, 2018).

\subsection{Forecast Generation and Evaluation}

To evaluate the forecasting accuracy of the presented methods, we use genuine out-of-sample forecasts. Each of the time series considered in the study contributes 730 observations to the sample. Because a sufficient amount of observations is needed to actually estimate an initial model, we reserve the first 90 time points as an introductory training set, while the remaining 640 observations are allocated to the initial test set. The forecast generation and evaluation scheme is illustrated in figure 2, where the black blocks represent observations that are incorporated in the model estimation, while grey and white circles in the boxes represent the generated forecasts. All forecasts that belong to the same forecast horizon are marked with the same color, e.g. the one-step-ahead forecasts shown in dark grey. In total, this cross-validation scheme yields 34,300 evaluable forecasts per method and time series pair. Given the 2,000 electronic consumer products and 16 considered model variants, we extract our findings from a basis of almost 1.1 billion evaluated predictions.

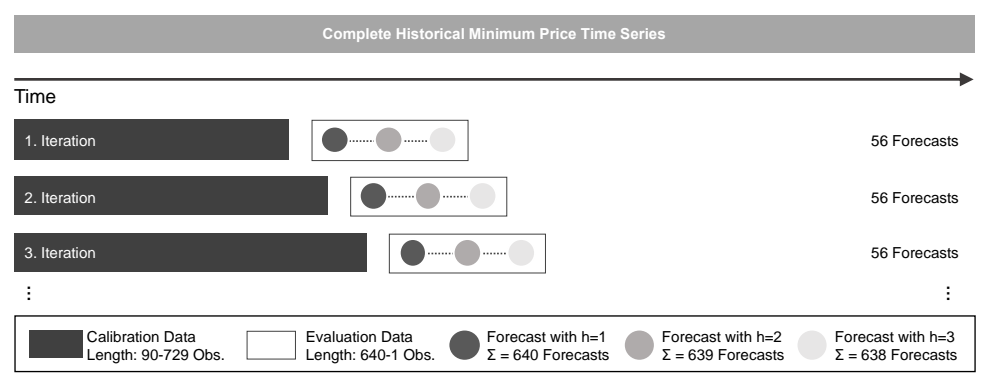

Figure 2. Rolling forecast evaluation scheme

Because we forecast prices, the generated predictions and their accuracy are related to the level of the respective time series. Thus, the need to make results comparable over all time points, forecast horizons, and products in the data set arises. For the evaluation, we, therefore, refer to the mean absolute percentage error (MAPE) per product as the main performance indicator. The MAPE for a specific forecast horizon $h$ conditional on a specific product and method is given by

$$
M A P E_{h}=\frac{1}{640-h+1} \sum_{k=1}^{640-h+1}\left|\frac{y_{90+h+k-1}-\hat{y}_{90+h+k-1}}{y_{90+h+k-1}}\right|
$$

For each method, the evaluation measure is calculated over all forecasts that have been generated for one horizon (e.g. for $h=1$ over the grey circles in figure 2). By being an aggregated measure, the MAPE allows 
gaining an understanding of the overall performance for the specific product and for different forecast horizons. We focus on presenting our findings for the traditional MAPE, which is not only more widely used but also allows comparability with other studies (Clements, 2005). However, results of other measures are consistent.

\section{RESULTS}

The following chapter presents overall performance as well as measures differentiated by product category for the different forecasting methods. As all performance differences between methods are highly significant due to the extremely large sample size, the following section does not present evidence and results from statistical tests for mean differences or predictive performance in general. In order to be able to visualize the results, the product-dependent evaluation measures have been condensed by averaging over all products in the sample or over the respective product category. The performance indicators for each method, therefore, represent 1.28 million in total respectively 320,000 forecasts per category for the first horizon.

Table 2 shows the resulting performance measure in percent for the 16 methods. When interpreting the MAPE, random walk, AutoARIMA, and EXS perform well, however, better performance is noted by logTheta, FourTheta, EXS dHolt, and Combi SHD. Interestingly, estimating the Theta method on a logarithmically transformed price series improves performance by $0.18 \%$ relative to the accuracy of the Theta model estimated on the nominal price values. The worst performances expectedly come from models that are not tied to the last observations of the series, so Mean as well as the linear trend, fail to deliver convincing results.

Table 2. Forecasting results in \%

\begin{tabular}{|c|c|c|c|c|c|c|c|c|c|c|c|c|c|c|c|c|}
\hline Met & Trend & \begin{tabular}{|l|} 
Trend \\
sq.
\end{tabular} & \begin{tabular}{|l} 
Trend \\
exp.
\end{tabular} & \begin{tabular}{|l|} 
Trend \\
90
\end{tabular} & Mean & $\begin{array}{l}\text { Mean } \\
90\end{array}$ & $R W$ & \begin{tabular}{|l|}
$R W$ \\
Drift
\end{tabular} & \begin{tabular}{|l|} 
Auto \\
ARIMA
\end{tabular} & \begin{tabular}{|l} 
Auto \\
ARIMA \\
inst. \\
\end{tabular} & $E X S$ & $\mid \begin{array}{l}E X S \\
d H o l t\end{array}$ & \begin{tabular}{|l|} 
Four \\
Theta
\end{tabular} & $\begin{array}{l}\text { Log } \\
\text { Theta }\end{array}$ & $\begin{array}{l}\text { Combi } \\
E A\end{array}$ & $\begin{array}{l}\text { Combi } \\
\text { SHD }\end{array}$ \\
\hline IAPE & 13.23 & 11.61 & 9.8 & 7.14 & 20.77 & 8.35 & 5.01 & 6.31 & 6.06 & 5.98 & 5.21 & 4.99 & 4.98 & 4.93 & 5.37 & 4.9 \\
\hline
\end{tabular}

Noteworthy insights are found when comparing models over time within their classes as shown in figure 3. As expected, the presented MAPE values increase with growing forecast horizons due to the rising uncertainty. This behavior is consistent throughout all methods. One should note that the value range of the axes between the first and second rows has been adapted to make results more legible. Figure 3 shows the forecast accuracy over a forecasting window from $h=1$ to 56 for all methods. While the global mean is, without a doubt, the worst method to choose, Theta and Combination models complemented by the random walk produce suitable forecasts and show similar courses of their graphs over the forecast horizons. Additionally, EXS dHolt performs well throughout the shown 56 forecast horizons and exhibits a MAPE below $8 \%$ at $h=56$ and by that delivers the third-best results after logTheta and Combi SHD and before FourTheta and RW. While the EXS and the ARIMA approaches deliver similar results for very short forecast horizons, ARIMA produces inferior results when horizons grow. This performance drift may explain why the combination of these two methodologies shows a deteriorated performance, while the combination of different EXS models (Combi SHD) delivers the second-best results at the end of the observation period.

Additionally, one should note that there are only a few crossing points between models and also between classes, meaning intersections, where it is favorable to switch from one method to another. In most cases, the performance order of the methods stays constant. An exception for that is the quadratic trend, which performs well for small forecast horizons, but its forecast error rises rapidly with increasing forecast horizons.

The results of trend models (3a) are very diverse compared to groups $3 \mathrm{c}$ and $3 \mathrm{~d}$. While the exponential and local (Trend 90) trend perform reasonably, the linear and quadratic trend appear to be not recommendable. Mean and random walk models ( $3 \mathrm{~b}$ ) also show very heterogeneous results with all random walk models outperforming the mean class. Within the random walk models, there is only a small difference between the $R W$ and $R W$ Drift in the short run. However, this quickly changes with increasing forecast horizons leading to highly deviating results - the random walk with drift being only the $10^{\text {th }}$-best model at $h=56$, while the normal random walk shows suitable performance for the price data. ARIMA models (3c) perform reasonably on rather short horizons in general; however, as figure 3c shows both EXS models outperform the ARIMA class. The very good performance of the automatic exponential smoothing procedure can be easily overlooked when compared to configuration options within its model class. This is because the Holt exponential smoothing method with a damped trend delivers the second-best results in the analysis of the average performance and 
third best when looking at $h=56$. As table 2 and figure $3 \mathrm{~d}$ show, FourTheta is slightly inferior to the logarithmized configuration of the original Theta model. Finally, Combi SHD consistently outperforms the combination of the automatic selection procedures of EXS and ARIMA.
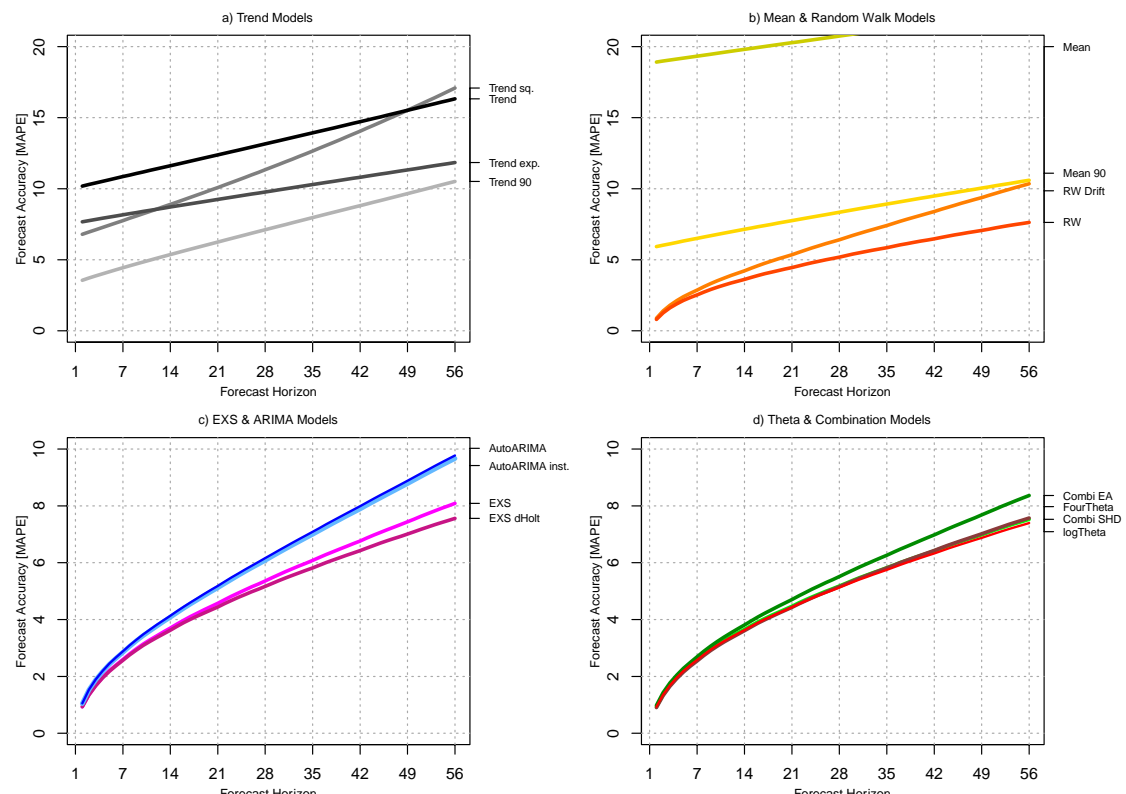

Figure 3. Result comparisons within model classes.

When discussing category-specific results, it is important to analyze which method delivers the best results for a given category. For refrigerators - from the 16 described methods - the FourTheta method delivers the best results directly followed by the random walk. Interestingly, the method including model adaptations with the lowest error is the single exponential smoothing model, which is the simplest form of exponential smoothing including only a weighted moving average and no components for trend or seasonality. For graphic cards, the pure $R W$ model works best for all 56 horizons. However, the $R W$ is narrowly outperformed by the FourTheta method for larger forecast horizons. The Combi SHD model delivers very good results for the smartphone product category. But pre- and postprocessing of the data in terms of applying the methods to logarithmic prices improves results significantly so that the logTheta method consistently outperforms all other methods independent of the forecast horizon. For the product category that is hardest to forecast - computer memory - Combi SHD works best overall. However, the Holt exponential smoothing model with a damped trend has very similar MAPEs resulting mainly from the good performance for larger forecast horizons.

All in all, it often seems to be advisable to transform the underlying data before generating predictions. Transformations like the exemplarily discussed logarithmic one, do not guarantee better performance but sometimes improve results and rarely lead to significantly worse outcomes.

\section{CONCLUSION AND PRACTICAL IMPLICATIONS}

In this paper, we presented an in-depth empirical analysis evaluating the forecasting performance of 16 methods for price prediction services based on a large sample of product price time series. We showed that univariate time series forecasting methods deliver suitable performance and that these methods are a viable option when planning and designing services, that support customers while buying consumer goods. By explicitly focusing on consumer goods prices, we help facilitate the transition towards data-driven services and dynamic business models for price aggregation sites and enable them to support customers to make better, more informed, less costly buying decisions.

Due to the large sample size and the variability of products and forecast horizons, the performance of particular forecasting configurations can be derived easily from our analysis. We showed that even though methods like Theta models perform well in a lot of cases, a one-fits-all solution does not exist - neither for 
every product category nor for every forecast horizon. When designing a recommendation service this should be taken into account and adapted depending on the application context.

Generally, we find that simple methods are hard to outperform, which is an indication that more complex approaches could not fully leverage the specific data characteristics. Thus, it would be interesting to investigate which data features contribute to forecast accuracy.

\section{REFERENCES}

Assimakopoulos, V., Nikolopoulos, K., 2000. The theta model: A decomposition approach to forecasting. Int. J. Forecast. $16,521-530$.

Bolton, R.N., Shankar, V., Montoya, D.Y., 2006. Recent Trends and Emerging Practices in Retailer Pricing, in: Krafft, M., Mantrala, M.K. (Eds.), Retailing in the 21 st Century - Current and Future Trends. Springer, Germany, pp. 255-269.

Box, G.E.P., Cox, D., 1964. An analysis of transformations. J. R. Stat. Soc. - Ser. B. 26, 211-252.

Box, G.E.P., Jenkins, G.M., Reinsel, G.C., 1994. Time Series Analysis: Forecasting \& Control. Wiley, Hoboken, NJ.

Buchwitz, B., Küsters, U., 2018. Should I buy my new iPhone now? Predictive Event Forecasting for Zero-Inflated Consumer Goods Prices. Proc. 39th Int. Conf. Inf. Syst. 1-16.

Clemen, R.T., 1989. Combining forecasts: A review and annotated bibliography. Int. J. Forecast. 5, 559-583.

Clements, M.P., 2005. Evaluating Econometric Forecasts of Economic and Financial Variables. Palgrave Macmillan, NY.

Gardner, E.S., 2006. Exponential Smoothing: The State of the Art - Part II. Int. J. Forecast. 22, 637-666.

Gardner, E.S., 1985. Exponential Smoothing: The State of the Art. J. Forecast. 4, 1-28.

Holt, C.C., 2004. Forecasting seasonals and trends by exponentially weighted moving averages. Int. J. Forecast.

Hyndman, R.J., Athanasopoulos, G., 2018. Forecasting: Principles and Practice, 2nd ed. OTexts.

Hyndman, R.J., Billah, B., 2003. Unmasking the Theta method. Int. J. Forecast. 19, 287-290.

Hyndman, R.J., Khandakar, Y., 2008. Automatic time series forecasting: The forecast package for R. J. Stat. Softw. 27, $1-22$.

Hyndman, R.J., Koehler, A.B., Ord, K., Snyder, R.D., 2008. Forecasting with Exponential Smoothing - The State Space Approach. Springer, Berlin, Germany.

idealo, 2017. Bestseller und ihr Preisverfall - Wann klingelt der Preiswecker. Url: https://www.idealo.de/magazin/2017/ 07/31/bestseller-und-ihr-preisverfall-wann-klingelt-der-preiswecker/.

Kannan, P.K., Kopalle, P.K., Åsbrink, S., 2001. Dynamic Pricing on the Internet: Importance and Implications for Consumer Behavior. Int. J. Electron. Commer. 5, 63-83.

Kömm, H., Küsters, U., 2015. Forecasting zero-inflated price changes with a Markov switching mixture model for autoregressive and heteroscedastic time series. Int. J. Forecast. 31, 598-608.

Kopalle, P., Biswas, D., Chintagunta, P.K., Fan, J., Pauwels, K., Ratchford, B.T., Sills, J.A., 2009. Retailer Pricing and Competitive Effects. J. Retail. 85, 56-70.

Levy, M., Grewal, D., Kopalle, P.K., Hess, J.D., 2004. Emerging trends in retail pricing practice: Implications for research. J. Retail. 80, XIII-XXI.

M4 Competition, 2018. Competitor's Guide. University of Nicosia, Url: https://www.m4.unic.ac.cy/wp-content/uploads/ 2018/03/M4-Competitors-Guide.pdf

Makridakis, S., Hibon, M., 2000. The M3-Competition: results, conclusions and implications. Int. J. Forecast. 16, 451-476.

Makridakis, S., Wheelwright, S.C., Hyndman, R.J., 2008. Forecasting Methods and Applications, 3rd ed. Wiley India, New Delhi.

Nikolopoulos, K., Assimakopoulos, V., 2004. Generalizing the Theta model for automatic forecasting. Int. Symp. Forecast. ISF 2004, Sydney, Australia.

Pan, X., Ratchford, B.T., Shankar, V., 2002. Why Aren't the Prices of the Same Item the Same at Me. Com and You.Com?: Drivers of Price Dispersion Among E-Tailers. SSRN 328820.

Pegels, C.C., 1969. Exponential Forecasting: Some New Variations. Manage. Sci. 15, 311-315.

Rydberg, T.H., Shephard, N., 2003. Dynamics of Trade-by-Trade Price Movements: Decomposition and Models. J. Financ. Econom. 1, 2-25.

Sucarrat, G., Grønneberg, S., 2016. Models of Financial Return with Time-Varying Zero Probability. MPRA Work. Pap. $1-25$.

Winkelmann, R., 2008. Econometric Analysis of Count Data, 5th ed. Springer, Germany. 